\title{
Neurosarcoidosis Diagnosed After Three Decades of Evolution A Case of Perseverance and Insight
}

\author{
Jason L. Jia ${ }^{1}$, Catherine Maurice ${ }^{2 *}$ \\ ${ }^{1}$ Faculty of Medicine, MD Candidate Class of 2019, University of Toronto, Ontario, Canada. \\ ${ }^{2}$ Department of Medicine, Division of Neurology, Princess Margaret Cancer Centre, University Health Network, University of Toronto, Ontario, \\ Canada. \\ * Corresponding author: Catherine Maurice, Department of Medicine, Division of Neurology, Princess Margaret Cancer Centre, University \\ Health Network, University of Toronto, Ontario, Canada. \\ Email: Catherine.Maurice@uhn.ca \\ Received date: December 14, 2019; Accepted date: December 22, 2019; published date: December 30,2019 \\ Citation: Jason LJ, Catherine M (2019) Neurosarcoidosis Diagnosed After Three Decades of Evolution A Case of Perseverance and Insight. J \\ Clinical Research and Reports, 1(1); DOI:10.31579/jcrr.19/007 \\ Copyright: (c) 2019 Catherine M. This is an open access article distributed under the Creative Commons Attribution License, which permits \\ unrestricted use, distribution, and reproduction in any medium, provided the original work is properly cited.

\begin{abstract}
A lymphoma survivor presented for reassessment of his long-standing neurological symptoms more than three decades following a diagnosis of systemic T-cell lymphoma. Despite our desire to establish the diagnosis promptly, time is often necessary to reach conclusions based on the patient's clinical evolution. We recount the chronology of clinical and radiological findings that, until recently, were largely inexplicable. We then report on the challenging diagnosis of neurosarcoidosis and suggest a known, yet rare, continuity linking it with lymphoma. Finally, we emphasize on the clinical evolution as a determining factor in the quest for a complex diagnosis. Insight, judgment, and perseverance are featured as key virtues of a resilient Neurologist.
\end{abstract}

Key Words: sarcoidosis, neurosarcoidosis; t-cell lymphoma; anti-tr paraneoplastic syndrome; granulomatous disease

\section{Case Report}

In 1990, a 31-year-old male known for thoracic T-cell lymphoma presented for new-onset ataxia. He underwent chemotherapy and achieved remission within a year. Six years after oncological presentation, this gentleman developed pancerebellar symptomatology. A brain MRI revealed numerous bi-hemispheric white matter lesions consistent with lymphoma recurrence to the CNS, surprisingly sparing the posterior fossa, thus a paraneoplastic cerebellar syndrome $(\text { anti-Tr })^{1}$ was suspected. The patient was treated with prednisone according to the clinical presentation, in an era of laboratory limitations for paraneoplastic antibodies. Following the curative treatment of the underlying neoplasia and steroid exposure, his symptoms resolved. Unexpectedly, several attempts to taper the oral prednisone failed over the subsequent three decades, leading to deleterious complications from prolonged steroid exposure.

Based on this patient's medical history, failure of steroid tapering was unjustified, and we were motivated to revisit every detail. We reattempted an extremely slow tapering of oral prednisone, decreasing the initial dose of $60 \mathrm{mg}$ OD by $2.5 \mathrm{mg}$ every two weeks. The purpose was to document the nature of the previous failures, considering the possibility of asthenia due to adrenal insufficiency versus focal neurologic symptoms due to distinct CNS lesions. At half of the prednisone daily dose, the patient developed fatigue, dysphagia, and diffuse pyramidal weakness. Concomitant respiratory distress associated with pleural thickening, lobar consolidation and PET-FDG uptake further complicated the picture. Clinical examination was hindered by severe steroid-induced proximal muscular atrophy confining our patient to a wheelchair. Orthopedic procedures were required, resulting in a significant deconditioning affecting his global function.

A brain MRI showed numerous subacute enhancing white matter lesions scattered throughout the white matter of bilateral cerebral hemispheres (Figure 1) 


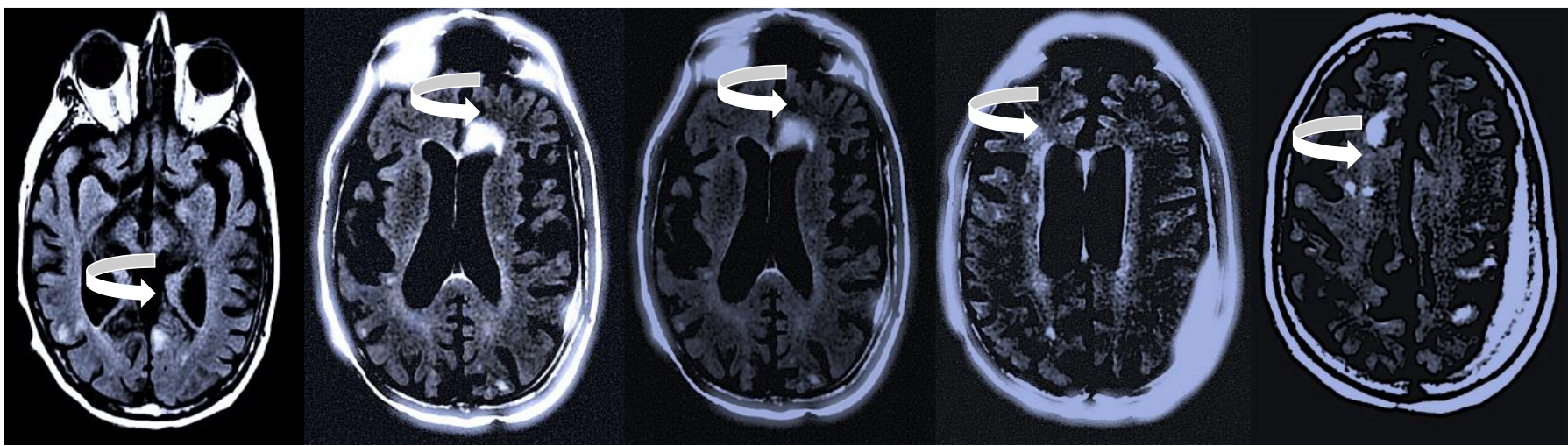

CAUDAL
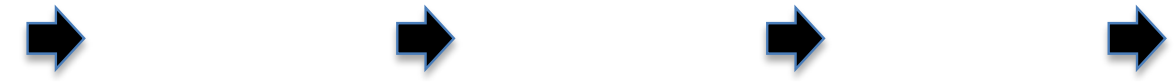

CRANIAL

\section{Figure 1: Neurosarcoidosis is a condition among many others, associated with multiple bihemispheric hyperintense white matter lesions.}

Legend: Brain MRI, unenhanced axial FLAIR sequences represented caudal (respectively from left to right) demonstrating multifocal patchy white matter hyperintensities scattered throughout bilateral hemispheres and associated with pachymeningeal thickening.

Thick arrows: Showing the progression of MRI sequences "CAUDAL CRANIAL".

Small Curved Arrows:

Pointing at examples of white matter lesions.

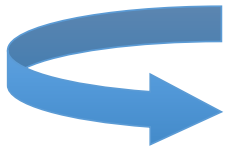

Those T2/FLAIR hyperintense lesions were accompanied by diffuse pachymeningeal thickening. The differential diagnosis included: CNS lymphoma recurrence, granulomatous disease, opportunistic infections, as well as a paraneoplastic syndrome relapse. Opportunistic infections are linked to severe and prolonged immunosuppression, whereas we were in the process of restoring his immune function. Paraneoplastic syndromes appear concomitantly to the onset or recurrence of an underlying neoplasia, which was unsuspected in our case considering the cure of his T-cell lymphoma, several years prior ${ }^{2-3}$. There was no evidence of a new primary cancer in development. Steroids are renowned for their cytotoxic properties on lymphomatous cells, impacting the aspect of white matter lesions following steroid exposure ${ }^{4-5}$. This was the case for our gentleman, whose brain biopsy was postponed due to the disappearance of hemispheric lesions following prednisone administration. However, despite the cytotoxic property of steroids, the likelihood of controlling an active CNS lymphoma over three decades with oral prednisone monotherapy is extremely low. Considering the development of respiratory symptoms concomitant to the neurologic deterioration, a unifying granulomatous disease was our leading hypothesis.

Our patient finally underwent a cerebral biopsy involving an active lesion and the adjacent dura. The pathology revealed a reactive lymphocytic infiltrate without monoclonal population or infectious organism, compatible with an inflammatory process. Retrospectively, a review of historical records revealed a chest X-Ray from the 1980s, performed in another context, incidentally showing pulmonary opacities, compatible with sarcoidosis. Moreover, the multisystemic symptoms improved a few months following the onset of azathioprine. This validated our suspicion that the patient's neurological deterioration and peculiar sensitivity to prednisone was attributed to neurosarcoidosis.

The co-occurrence of lymphoma and sarcoidosis is a rare and intriguing phenomenon described as the sarcoidosis-lymphoma syndrome $^{6}$. It is theorized that granulomatous lesions act as a microenvironment of aberrant immune function. Elevated CD4+ activity increases the risk of developing dysplastic changes, while diminished CD8+ presence impairs immunoregulatory capacity. Unrestricted T-cell activation is promoted, ultimately leading to lymphoma transformation. We have presented the work-up of a steroid-dependant neurologically impaired lymphoma survivor leading to the diagnosis of neurosarcoidosis. This case exemplifies the fundamental and variable relationships between lymphoma and its numerous comorbidities. Furthermore, we demonstrate that the manifestations of cancer may be represented in figurative balance oscillating between immunosuppression and immunoreactivity, looking for its equilibrium. Finally, this case emphasizes the basic tenet of neurological investigation: do not dismiss clinical evolution or leave any stone unturned in challenging or inexplicable circumstances; the answer is often in front of us, buried under a pile of confounding factors.

\section{Acknowledgement}

Acknowledgement to Dr. Warren P. Mason for mentorship of Dr. Catherine Maurice when this patient presented initially at Princess Margaret Hospital, Toronto, ON, Canada.

\section{Authors Contributions}

Dr. Jason Jia was responsible for conceptualization, redaction, editing, and revision.

Dr. Catherine Maurice was responsible for conceptualization, redaction, editing, figures, supervision and revision.

\section{Disclosures}

No sponsorship was received for the redaction of this manuscript.

The authors did not receive any financial compensation.

The authors did not receive any research support.

The authors do not have any stocks, stock options or royalties in the medical industry.

The authors are not involved in legal proceedings.

Dr Jason Jia has no disclosures.

Dr Catherine Maurice has no disclosures. 


\section{References}

1. Smitt PS, Graaff E, Maat P, Hulsenboom E, van den Berg R, et al (2012) Identifying the Anti-Tr Antigen in Paraneoplastic Cerebellar Degeneration. Neurology; 78(1):S45.002.

2. Pelosof LC, Gerber DE (2010) Paraneoplastic Syndromes: An Approach to Diagnosis and Treatment. Mayo Clin Proc;85(9):838854.
3. Honnorat J, Antoine JC (2007) Paraneoplastic neurological syndromes. Orphanet J Rare Dis;2:22.

4. Smith LK, Cidlowski JA (2010) Glucocorticoid-induced apoptosis of healthy and malignant lymphocytes. Prog Brain Res;182: 1-30.

5. Lin KT, Wang LH (2016) New dimension of glucocorticoids in cancer treatment. Steroids; 111: 84-88.

6. Brincker H (1986) The sarcoidosis-lymphoma syndrome. Br J Cancer; 54(3):467-473. 